\title{
Thermal property changes of poly( $N$-isopropylacrylamide) microgel particles and block copolymers
}

\author{
Klaus Tauer • Daniel Gau • Susanne Schulze • \\ Antje Völkel $\cdot$ Rumiana Dimova
}

Received: 30 October 2008 /Revised: 8 December 2008 / Accepted: 8 December 2008 /Published online: 3 January 2009

(C) The Author(s) 2008. This article is published with open access at Springerlink.com

\begin{abstract}
Investigation of the thermo-reversible properties of different poly( $N$-isopropyl acrylamide) samples, including microgels and block copolymers, with a combination of methods such as electron microscopy, dynamic light scattering, analytical ultracentrifugation, electrophoresis and ultrasound resonator technology allows comprehensive characterisation of the phase transition. By the combination of methods, it was possible to show that the precipitated polymer phase contains at $40{ }^{\circ} \mathrm{C}$ between 40 and 50 vol.\% of water. Besides free bulk water, there is also bound water that strongly adheres to the $N$-isopropyl acrylamide units (about 25 vol.\%). Ultrasound resonator technology, which is a non-sizing characterisation method, revealed for the microgel particles two more temperatures (at about 35 and between $40{ }^{\circ} \mathrm{C}$ and $50{ }^{\circ} \mathrm{C}$ depending on the chemical nature) where characteristic changes in the ultrasound attenuation take place. Moreover, the experimental data suggest that the phase transition temperature is related to surface charge density of the precipitated particles.
\end{abstract}

Keywords PNIPAM microgels and block copolymers . Characterisation of thermal properties

\footnotetext{
K. Tauer $(\triangle) \cdot$ A. Völkel $\cdot$ R. Dimova

MPI Colloids and Interfaces,

14476 Golm, Germany

e-mail: Klaus.Tauer@mpikg-golm.mpg.de

D. Gau $\cdot$ S. Schulze

TF Instruments $\mathrm{GmbH}$,

Im Neuenheimer Feld 583,

69120 Heidelberg, Germany
}

\section{Introduction}

Since the first report of a lower critical solution temperature (LCST) of poly( $N$-isopropylacrylamide) (PNIPAM) in a scientific paper, 40 years ago [1], and subsequent discoveries that by the addition of surfactants [2] or by copolymerisation with acrylamide and cross-linkers [3] thermo-sensitive colloidal particles are accessible, the polymer became most popular as a kind of standard material. The precipitation temperature of PNIPAM in diluted solutions is about $32{ }^{\circ} \mathrm{C}$ (plus or minus a few degrees depending on the particular conditions), which is an attractive range for biomedical applications, particularly drug delivery [4-8]. Hence, quite a large number of reviews have been published until now [3,9-15]. This temperature is frequently, also in this contribution, denoted as LCST. However, in a strict thermodynamic sense, it is a precipitation and dissolution temperature upon heating and cooling, respectively. The miscibility gap of PNIPAM has a minimum at weight fraction between 0.4 and 0.5 (slightly depending on the molecular weight of the sample) at a temperature, the actual LCST, between $26^{\circ} \mathrm{C}$ and $27{ }^{\circ} \mathrm{C}$. Only in very diluted or very concentrated solutions does the phase separation take place at higher temperatures [16]. Other experimental results confirm a minimum in the temperature-concentration phase diagram at about $50 \mathrm{wt} . \%$ but at temperature below $25^{\circ} \mathrm{C}$ [17].

The thermo-reversible behaviour of PNIPAM depends strongly on the architecture of the macromolecules such as the kind of comonomers leading to random or block copolymers $[10,15,18-26]$ but also on the nature of the endgroups [27-34]. Though PNIPAM is quite a long time in the focus of the research, a lot of interesting results appear still each year. The following examples should 
illustrate this. Recently, $N, N$-diethylacrylamide-co- $N$ isopropylacrylamide copolymer microgel particles have been described, showing a synergistic depression of the transition temperature as the phase transition takes place at temperature lower than that of the corresponding homopolymers [35]. Thermo-responsive PNIPAM shells around hydrophobic cores that contain inorganic nanoparticles are interesting inorganic polymeric composite materials for various potential applications. The synthesis of hybrid core-shell particle with about 33 wt. $\%$ of magnetic nanoparticles in the core is reported in [36]. The application of siloxane comonomers is a new and innovative approach to cross-link the particles via hydrolysis-condensation reaction and an alternative route to inorganic organic hybrid materials [37].

In summary, the LCST of PNIPAM is not a single value characterising the polymer per se unambiguously but is much more the result of rather complex interactions taking place over a certain temperature range and depends for a given solvent on the polymer concentration, the molecular weight, the nature of the end groups and the comonomer content and distribution along the chain. For chemists, the possibility to tune the phase separation by the molecular architecture is a truly fascinating challenge.

Reported in this contribution are results of a comprehensive study of the thermo-reversible properties of chemically and morphologically different PNIPAM samples by means of scanning and transmission electron microscopy (SEM, TEM), dynamic light scattering (DLS), ultrasound resonator technology (URT), analytical ultracentrifugation (AUC) and zeta potential measurements. Particularly PNIPAM microgels with anionic (PNIPAM-xl-A, sulfate endgroup), cationic (PNIPAM-xl-C, amidinopropane hydrochloride end group) and poly(ethylene glycol) (PNIPAM-xl-PEG, molecular weight of the PEG $2 \times 10^{4} \mathrm{~g} / \mathrm{mol}$ ) endgroups and poly(styrene sulfonate)-PNIPAM block copolymers (PSS-PNIPAM) were studied. Thermo-sensitive properties of PNIPAM-xl-PEG microgels and PNIPAM-PSS block copolymers are reported for the first time. On the one hand, the data allow conclusions regarding the influence of the state before the phase transition - either swollen particles (PNIPAM-xl-A, PNIPAM-xl-C, PNIPAM-xl-PEG) or dissolved single polymer molecules (PNIPAM-PSS). On the other hand, the experimental results prove the influence of the nature of the end groups incorporated during the polymerisation procedure or the molecular architecture. In all cases, two component systems are studied consisting of the particular PNIPAM sample and pure water. There is no surfactant to stabilise the colloidal particles because its presence influences at least the size of the precipitating particles. Moreover, it is known that surfactants, such as sodium dodecyl sulphate, are able to solubilise not only organic liquids but also polymer molecules in water $[32,38,39]$.
The applied analytical techniques enable conclusions regarding the influence of the detailed chemical nature on the phase transition. Particularly, results are reported regarding the reversibility of the precipitation/redissolution process, the morphology of the precipitate, the charge density and the self-stabilisation of the particles and, to the best of our knowledge, for the first time, the amount of hydration water bound strongly to the PNIPAM chain. Note that the hydration water is different from the amount of water present in the PNIPAM particles at T $>$ LCST, which is between 30 and $20 \mathrm{wt} . \%$ at temperatures between $35^{\circ} \mathrm{C}$ and $50{ }^{\circ} \mathrm{C}$ [5]. Moreover, by means of URT, other transitions of the PNIPAM microgel particles in the temperature range above the LCST of about $32{ }^{\circ} \mathrm{C}$ have been detected.

\section{Experimental information}

Materials NIPAM (Acros) was recrystallised from a mixture of hexane/toluene $(3 / 1, v / v)$, sodium styrene sulfonate from Sigma-Aldrich, poly(ethylene glycol) with a molecular weight of $20,000 \mathrm{~g} / \mathrm{mol}$ (Fluka), $N, N^{\prime}$-methylenebis (acrylamide) (MBA) from Fluka, ethyleneglycoldimethacrylate (EGDMA) from Aldrich, potassium peroxodisulfate (Fluka), 2,2'-azobis(2-amidinopropan)dihydrochlorid (V50) and 2,2'-azobis (2-methyl- $N$-(2-hydroxyethyl) propionamide (VA-086) both from Wako, ceric ammoniumchloride (CAN) from Fluka which were all used as received. Polymerisation and characterisation was carried out in ultrapure water (Seral purification system PURELAB Plus $^{\mathrm{TM}}$ ) with a conductivity of $0.06 \mu \mathrm{S} \mathrm{cm}^{-1}$.

Polymerisation The PSS precursor polymer was synthesised with the following recipe at $70{ }^{\circ} \mathrm{C}: 100 \mathrm{~g}$ of water, $20 \mathrm{~g}$ of sodium styrene sulfonate and $0.32 \mathrm{~g}$ VA-086. After polymerisation, the reaction mixture was extensively cleaned by ultrafiltration through DIAFLO membranes with a molecular weight cutoff of $10^{4} \mathrm{~g} / \mathrm{mol}$ (type YM 10 from Amicon, USA), and the polymer was isolated by freeze drying. The PNIPAM polymers were prepared by radical polymerisation at $70{ }^{\circ} \mathrm{C}$, except PNIPAM-PEG at $60{ }^{\circ} \mathrm{C}$, in all-glass reactors equipped with a jacket for heating or cooling to adjust the temperature, a condenser, a nitrogen inlet and outlet, a stirrer and a bottom valve to remove the reaction mixture. The reactor lid contains an additional opening for injecting the initiator solution after thermal equilibration of the reaction mixture. After polymerisation (polymerisation time 20-24 h), all polymers were cleaned by ultrafiltration through DIAFLO membranes with a molecular weight cutoff of $10^{5} \mathrm{~g} / \mathrm{mol}$ (type YM 10 from Amicon) as long as the amount of original water was replaced ten times. Then, the polymers were 
isolated by freeze drying. The polymerisation recipes of polymer samples are detailed in Table 1.

Characterisation Elemental analysis was carried out with Vario micro Cube, (elementar, Hanau, Germany). The chemical composition of PNIPAM-PEG and PNIPAMPSS was characterised oxygen and sulfur elemental analysis data, respectively. The molecular weight distributions of the PSS precursor polymer was analysed by analytical ultracentrifugation according to standard procedures [40, 41]. Some samples were investigated with electron microscopy after suspension preparation (either SEM with a LEO, ElectronMicroscopy, UK or TEM with a Zeiss EM 912 Omega microscope operating at $100 \mathrm{kV}$ ). Fourier transform infrared spectra were recorded with a Varian 1000 FT-IR spectrometer (Varian, USA) with a MIRacle ATR cell (Pike) between 600 and 4,000 1/cm. Dynamical differential scanning calorimetry (DSC) was performed with a DSC 204 (Netzsch, Germany) and glass transitions and melting points data are given for heating cycles.

For all characterisations of solutions or dispersions of the polymer samples, a stock solution/dispersion with ultrapure distilled water was prepared. The dilution procedure is detailed for the PSS-PNIPAM block copolymer. The block copolymer was dissolved in ultrapure distilled water to result in a 0.42 wt. $\%$ solution. This concentration gives a signal during the URT measurements whilst passing the LCST that can be nicely evaluated. The stock solution was repeatedly diluted until measurement of the average particle size above the LCST with routine dynamic light scattering equipment (Nicomp 370, Santa Barbara, USA) was still possible. The final solids content for dynamic light scattering was estimated to be $0.014 \%$ by weight. This low concentration guaranteed that the dynamic light scattering measurements are not influenced by interactions between the precipitated block copolymer particles. The final solids content was in any case below $1 \mathrm{wt} . \%$.

The particle size $\left(D_{\mathrm{i}}\right.$, intensity weighted average particle size) was measured by dynamic light scattering using a NICOMP particle sizer (either model 370 or 380) at temperatures between $25{ }^{\circ} \mathrm{C}$ and $60{ }^{\circ} \mathrm{C}$.

The change of the ultrasound velocity and attenuation was measured with the ResoScan ${ }^{\circledR}$ URT System (TF Instruments GmbH, Heidelberg, Germany) based on URT. This instrument is equipped with ultrasonic transducers made of lithium niobate single crystals with a fundamental frequency of $10 \mathrm{MHz}$. It contains twin sample cells for sample and reference with a path length of $7 \mathrm{~mm}$. The measurement volume is $200 \mu \mathrm{l}$. The temperature stability is $\pm 0.0003 \mathrm{~K}$ and the resolution of the ultrasonic velocity is $0.001 \mathrm{~m}^{-1} \mathrm{~s}^{-1}$. The ultrasound properties of the sample solutions are evaluated in relation to a highly diluted surfactant solution (ResoStandard, TF Instruments $\mathrm{GmbH}$ ) that practically does not alter the properties of pure water. All data reported are averages of at least three repeats. The evaluated signal of URT is either $\Delta U$ (ultrasound velocity) or $\Delta A$ (ultrasound attenuation) where the delta sign refers to the difference between the sample solution and the standard.

The sedimentation experiments have been performed on an Optima XLI centrifuge (Beckman Coulter, Palo Alto, CA, USA) and Rayleigh interference optics at $25^{\circ} \mathrm{C}, 32^{\circ} \mathrm{C}$ and $40{ }^{\circ} \mathrm{C}$ in pure water and $\mathrm{D}_{2} \mathrm{O}$-water mixture. The density of the continuous phase is calculated according to the actual dilution with $\mathrm{D}_{2} \mathrm{O}$ which is $1: 10$ except for sample PNIPAM-xl-B (1:30). Depending on the sedimentation velocity of the particles, speeds of 2,000, 3,000, 50,000 and $60,000 \mathrm{rpm}$ have been chosen to get an optimal resolution. The distributions of the sedimentation coefficients have been evaluated with the software SEDFIT (version 10.09 beta P. Schuck 2007) [42]. The density gradients have been performed on an Optima XLI centrifuge (Beckman Coulter) in methanol-bromoform mixtures $(75: 25, v / v)$ with absorbance optics at $500 \mathrm{~nm}$ and a speed of $40,000 \mathrm{rpm}$. The data have been evaluated with the program Newgradient (Kristian Schilling 2005, Nanolytics, Potsdam, Germany) [43].

Electrophoretic mobilities and particle size distributions at temperatures between $25^{\circ} \mathrm{C}$ and $50^{\circ} \mathrm{C}$ were determined with a Zetasizer Nano ZS (Malvern Instruments, Worcestershire, UK) operating with a $4 \mathrm{~mW} \mathrm{HeNe}$ laser $(632.8 \mathrm{~nm})$, a detector positioned at the scattering angle of $173^{\circ}$ and a temperature-control jacket for the cuvette. Each sample was degassed for $15 \mathrm{~min}$ to remove air bubbles. The cuvette was sealed to avoid evaporation and left for $5 \mathrm{~min}$ to allow temperature equilibration. Five measurements consisting of up to 12 consecutive runs of duration of $10 \mathrm{~s}$ were performed for each sample and temperature. Dynamic correlation functions were fitted by a second-order

Table 1 Polymerisation recipes for PNIPAM samples with various architectures

\begin{tabular}{lcclcc}
\hline Polymer & $\mathrm{H}_{2} \mathrm{O}(\mathrm{g})$ & NIPAM $(\mathrm{g})$ & Initiator/Oxidant & Precursor & Cross-linker \\
\hline PNIPAM-xl-A & 1,500 & 36 & $0.54 \mathrm{~g} \mathrm{KPS}$ & - & $1.77 \mathrm{~g} \mathrm{MBA}$ \\
PNIPAM-xl-C & 1,500 & 33 & $0.486 \mathrm{~g} \mathrm{~V} 50$ & - & $2 \mathrm{~g} \mathrm{MBA}$ \\
PNIPAM-xl-PEG & 1,455 & 23.7 & $6 \mathrm{~g} \mathrm{CAN} \mathrm{in} 70 \mathrm{~g} \mathrm{HNO}_{3}$ & PEG & $1.2 \mathrm{~g} \mathrm{EGDMA}$ \\
PNIPAM-PSS & 100 & 4 & $0.4 \mathrm{~g} \mathrm{CAN} \mathrm{in} 5 \mathrm{~g} \mathrm{HNO}_{3}$ & PSS \\
\hline
\end{tabular}


cumulant method to obtain the size distributions. For the zeta potential measurements, the samples (with volume $0.75 \mathrm{ml}$ ) were loaded in folded capillary zeta potential cells with integral gold electrodes. Three measurements consisting of 40 to 50 runs with duration of $10 \mathrm{~s}$ were performed. The mobility $\mu$ was converted to $\zeta$ potential using the Helmholtz-Smoluchowski relation $\zeta=\mu \eta / \varepsilon \varepsilon_{0}$, where $\eta$ is the solution viscosity, $\varepsilon$ the dielectric constant of water and $\varepsilon_{0}$ the permittivity in vacuum. The standard deviation is about $\pm 5 \mathrm{mV}$ as stated by the manufacturer.

\section{Results and discussion}

Sample characterisation

Samples PNIPAM-xl-A and PNIPAM-xl-C were prepared via common aqueous radical polymerisation of NIPAM in the presence of the water-soluble MBA cross-linker with potassium peroxodisulfate and 2,2'-Azobis(2-amidinopropan) dihydrochlorid as initiator, respectively. The resulting microgel particles have a quite high cross-linking density that allows easy re-dispergation of the purified and freezedried solids in water. The dispersed particles are stabilised by the corresponding ionic end groups stemming from the initiators and possess also at room temperature the typical milky white appearance of polymer latexes. Elemental analysis and Fourier transform infrared (FT-IR) spectroscopy give neither hints regarding the ionic endgroups nor the cross-linking units. DSC shows a glass transition temperature of $152{ }^{\circ} \mathrm{C}$ and $154{ }^{\circ} \mathrm{C}$ for PNIPAM-xl-A and PNIPAM-xl-C, respectively.

Sample PNIPAM-xl-PEG was prepared by aqueous radical polymerisation in the presence of the slightly water-soluble EGDMA cross-linker started with the redox initiation system ceric ion and poly(ethylene glycol) [44, 45]. The redox reaction between $\mathrm{Ce}^{4+}$ and the methylol endgroup leads under proton abstraction to a carbon radical and $\mathrm{Ce}^{3+}$. The cross-linked PNIPAM particles are stabilised by PEG chain ends and actually represent PNIPAM-PEG block copolymer microgel particles. Elemental analysis shows a ratio of about 8.8 ethylene glycol units per $N$-isopropylacrylamide unit $(N$ content is decreased to $2.8 \mathrm{wt} . \%$ ). FT-IR spectra confirm the presence of PEG due to strong absorption of the ether groups and the $\mathrm{CH}_{2}$ groups at about 1,100 and $2,9001 / \mathrm{cm}$, respectively, besides the typical absorption bands for PNIPAM. The sample can be re-dispersed in water after ultrafiltration and freeze drying without any problems. However, at similar solids content, the turbidity is at room temperature much lower compared to samples PNIPAM-xlA and PNIPAM-xl-C. This behaviour points to a lower cross-linking density of the PNIPAM core, which might be expected due to the lower water solubility of the EGDMA cross-linker and the start of the reaction with polymeric PEG radicals. The DSC data show no glass transition temperature of the cross-linked PNIPAM block but a melting peak at $65.6^{\circ} \mathrm{C}$, which is slightly higher than that observed for the starting PEG $\left(63.1^{\circ} \mathrm{C}\right)$.

The PNIPAM-PSS block copolymer was also prepared via aqueous radical redox polymerisation of NIPAM started with ceric ion and PSS precursor polymer with methylol endgroups as reductant. The molecular weight of the PSS precursor is between $10^{5}$ and $1.6 \times 10^{6} \mathrm{~g} / \mathrm{mol}$ (analytical ultracentrifugation). The nitrogen to sulphur ratio in the block copolymer is 4.6 (elemental analysis), and hence, a molecular weight between $3.2 \times 10^{5}$ and $5.1 \times 10^{6} \mathrm{~g} / \mathrm{mol}$ for the PNIPAM block can be estimated. Then, the overall molecular weight of the block copolymer is supposed to be between $4.2 \times 10^{5}$ and $6.7 \times 10^{6} \mathrm{~g} / \mathrm{mol}$. FT-IR spectra confirm the presence of PNIPAM and PSS units. At room temperature, the sample dissolves easily in water and forms a transparent solution. As the block copolymer is extremely hydrophilic, it contains practically always a few per cent of bound water also in the dry state. The DSC trace of the block copolymer shows a glass transition in the temperature range of about $130{ }^{\circ} \mathrm{C}$ which belongs to the PNIPAM block and a second peak in the range of $20^{\circ} \mathrm{C}$. The latter peak arise presumably from the PSS block, as high concentrated solutions of the homopolymer show in this range a temperature response which is attributed to the melting of spherulites [46].

Finally, these four samples represent three classes of PNIPAM structures: cross-linked microgel particles with different endgroups (PNIPAM-xl-A, PNIPAM-xl-C), block copolymer microgel particles (PNIPAM-xl-PEG) and linear block copolymers (PNIPAM-PSS).

Size and morphology of the dried samples—electron microscopy

It is to be expected that the morphological changes during the precipitation/redissolution of PNIPAM depend to large extent on the molecular architecture of the particular sample. Evaluation of light scattering data and zeta potential measurements require information on the shape of the particles. The assumption of spherical particles, though it is from the thermodynamic point of view quite reasonable, must not be valid in any case. The morphology of PNIPAM aggregates can considerably deviate from solid spheres especially in the dispersed state of non-cross-linked samples.

The morphology of PNIPAM-PAA block copolymers ( 2 wt.\% aqueous solution) with 74 NIPAM and 110 acrylic acid units was studied by means of cryogenic TEM below $\left(20^{\circ} \mathrm{C}\right)$ and above $\left(45^{\circ} \mathrm{C}\right)$ the LCST as well at $\mathrm{pH}$ values of 4.5 and 5.6 [23]. In any case, spherical objects have been observed. The size of the particles depends on the particular 
conditions and is the largest at $20{ }^{\circ} \mathrm{C}$ and $\mathrm{pH}=5.6$ (>300 $\mathrm{nm}$ in diameter with loosely packed outer region and solid core). At $\mathrm{pH}=4.5$ and $20{ }^{\circ} \mathrm{C}$ as well at $\mathrm{pH}=5.6$ and $45{ }^{\circ} \mathrm{C}$, the images show particles with diameters between 10 and $30 \mathrm{~nm}$. At poor solubility conditions for either block ( $\mathrm{pH}=4.5$ and $\left.45^{\circ} \mathrm{C}\right)$, solid particles of about $150 \mathrm{~nm}$ in diameter are detected.

The electron microscopy images put together in Fig. 1 show clearly solid spheres for the dried microgel particles (PNIPAM-xl-A, PNIPAM-xl-C). Moreover, the images prove the monodispersity of these samples. The enumeration of TEM pictures (only between 200 and 500 particles have been counted as the particle size distribution is quite monodisperse) resulted in the following average diameters for PNIPAM-xl-A: number average diameter $D_{\mathrm{n}}=486.8 \mathrm{~nm}$ and weight average diameter $D_{\mathrm{w}}=487.7 \mathrm{~nm}$, for PNIPA-xlC: $D_{\mathrm{n}}=455.2 \mathrm{~nm}$ and $D_{\mathrm{w}}=456.3 \mathrm{~nm}$.

In contrast, rather non-specifically shaped aggregates are found on TEM images of the microgel PNIPAM-xl-PEG (cf. Fig. 2).

The shape of the PNIPAM-PSS block copolymer precipitate is mainly spherical, however, with a quite broad size distribution ranging from below 50 to above $200 \mathrm{~nm}$ (cf. images of Fig. 3).

The similarity of the structures observed for PNIPAMPSS samples placed on the electron microscopy grid below and above the LCST seems reasonable for this highly diluted starting solution and proves that spherical block copolymer particles/micelles are formed within the biphasic region of the phase diagram under these particular conditions. Obviously, precipitation leads to similar morphologies whether the phase boundary is passed by increasing the temperature or the concentration. Additionally, smaller precipitation structures are visible, arising very likely from molecules that are not aggregated onto the larger particles.

Note that in contrast to PNIPAM-xl-A and PNIPAM-xl-C, both the morphology and the size of the other two samples are neither in the dissolved nor in the precipitated state clearly defined. This fact restricts the data evaluation of these samples as discussed below.

Size and morphology of the wetted samples - dynamic light scattering and analytical ultracentrifugation

Measurement of the hydrodynamic size of the thermoresponsive samples in dependence on temperature with routine dynamic light scattering equipment is quite common to characterise the LCST behaviour. Microgel particles (samples PNIPAM-xl-A, PNIPAM-xl-C, PNIPAM-xl-PEG) shrink during temperature increase, whereas soluble polymers (sample PNIPAM-PSS) precipitate and the apparent particle size increases. This principally different dependence is
Fig. 1 Electron microscopy images of PNIPA-xl-A (left hand) and PNIPAM-xl-C (right hand) after ultrafiltration and redispergation; TEM (upper row) and SEM images (lower row)
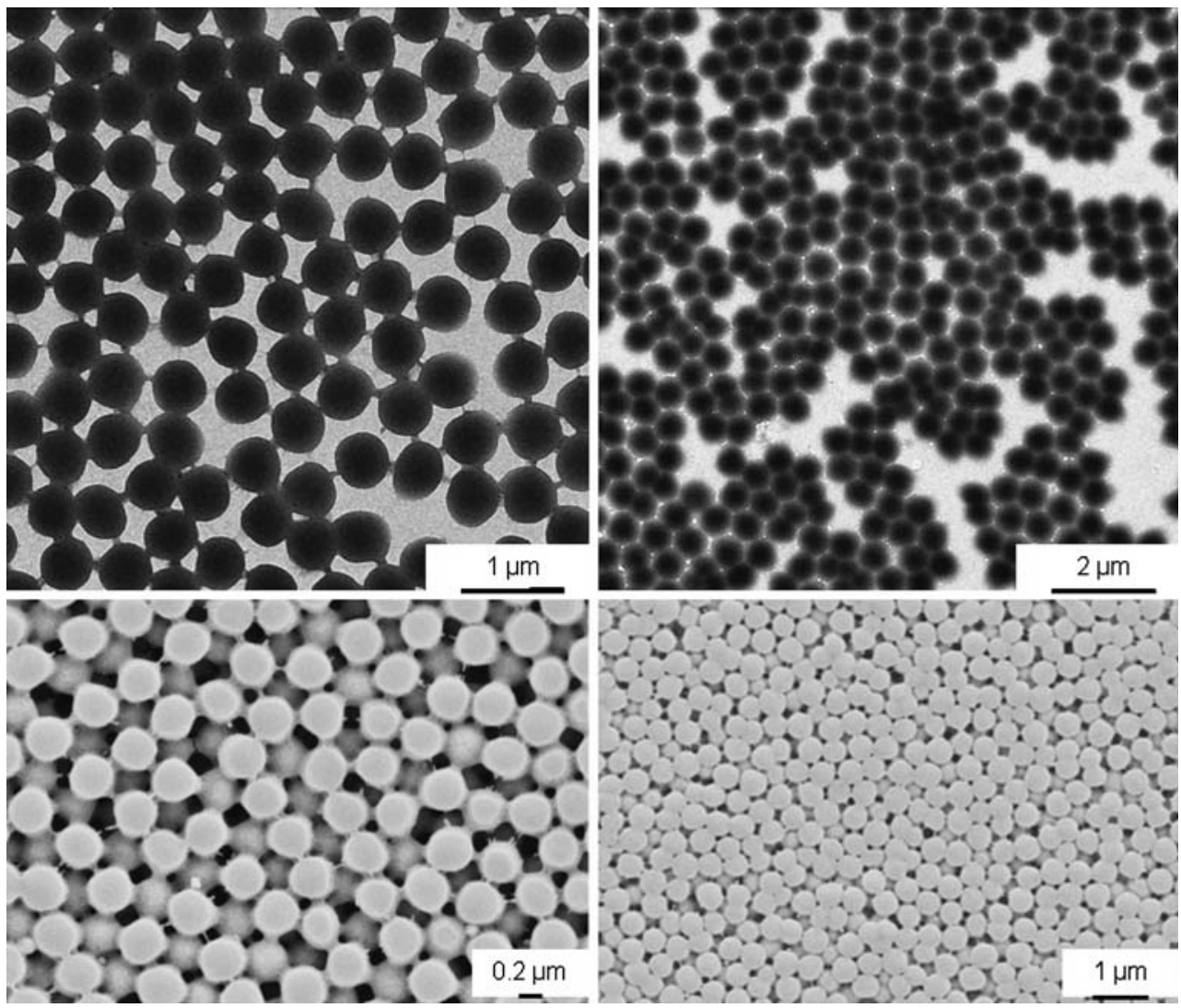
Fig. 2 TEM images of sample PNIPAM-xl-PEG

Fig. 3 TEM images of sample PNIPAM-PSS measured after preparation at room temperature (left hand) and at $40{ }^{\circ} \mathrm{C}$ (right hand)
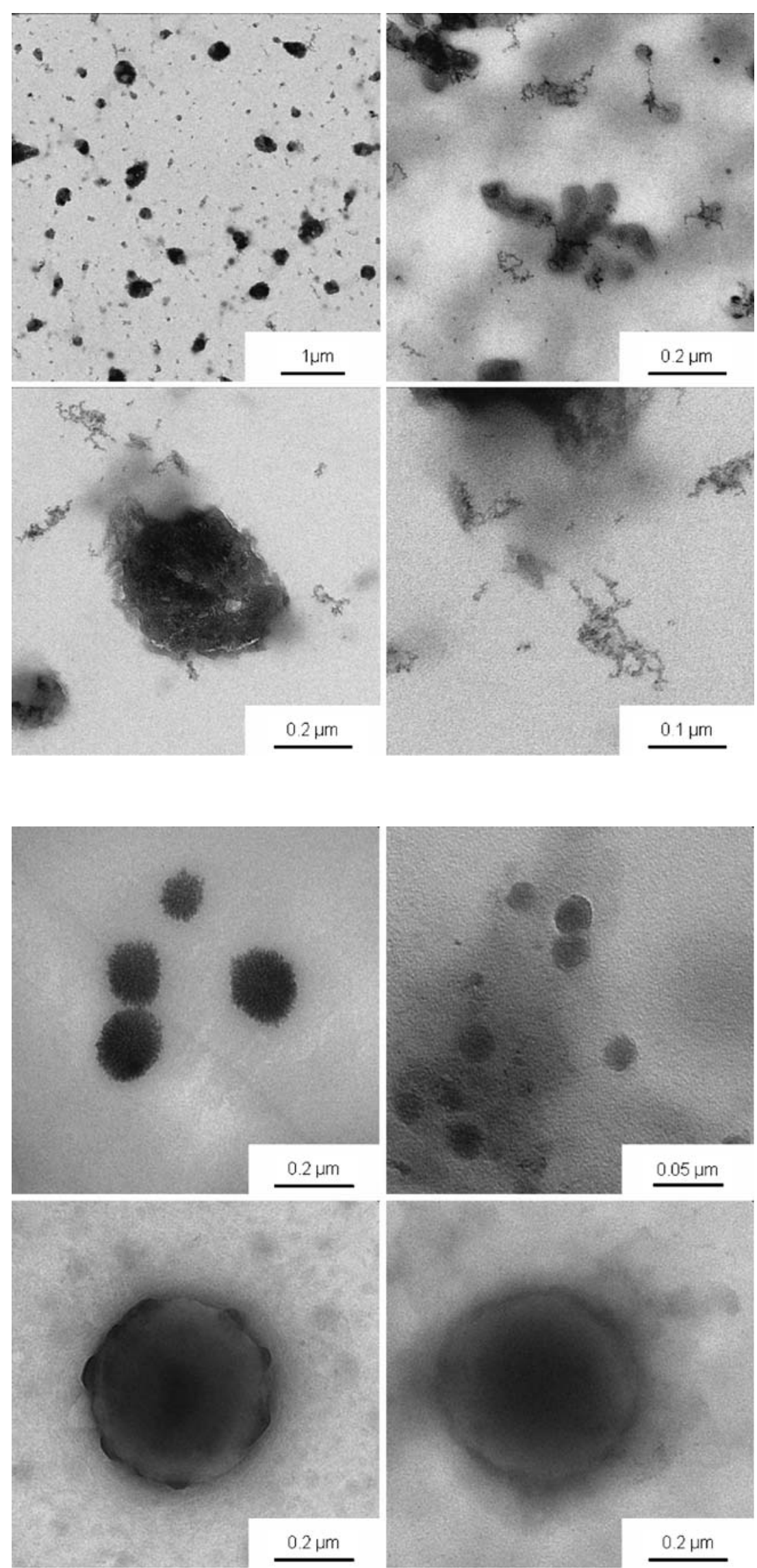
illustrated by the data put together in Fig. 4. The relative errors, determined from repeated heating and cooling cycles, indicate that the reproducibility for the higher cross-linked samples (PNIPAM-xl-A, PNIPAM-xl-C) is clearly better compared with the only loosely cross-linked PNIPAM-xlPEG or the block copolymer PNIPAM-PSS.

For the PNIPAM-PSS block copolymer, the difference in the heating and cooling curve indicates hysteresis. It is just a single point below the LCST during cooling, but it was repeatedly observed. Caution is recommended regarding the temperature during the measurements, as the there is no possibility to check the temperature for the particular light scattering equipment. However, the occurrence of hysteresis is not surprising as it is also observed for the coilglobule transition of single PNIPAM homopolymer chains as intensely studied by more sophisticated light scattering techniques with much higher sensitivity [47]. Contrary, the cross-linked particles do not show hysteresis but rather pulsating type of behaviour during temperature cycles. The different behaviour is reasonable, as the morphological changes during the redissolution of the PNIPAM-PSS particles require chain disintegration or disentanglement inside the multi-chain particles. The spring-like pulsation of the microgel particles requires only minor reorientation of macromolecules, but only the diffusion of water molecules in or out of the network.

The extent of swelling can be quantitatively expressed in various ways by means of particle sizes determined in the nonswollen (dry) or swollen (wet) state. The average diameters determined by enumerating TEM images correspond to the size of the dried particles $\left(D_{\text {dry }}\right)$, whereas the DLS values are that of the swollen or wet particles $\left(D_{\text {wet }}\right.$ or $\left.D_{i}\right)$. Thus, the swelling ratio $\left(H_{\mathrm{v}}\right)$ as defined by Eq. 1 or the volume fraction of water $\left(\phi_{\mathrm{w}}\right)$ are easily accessible, but reliable values can be obtained in this way only for the highly crosslinked monodisperse samples PNIPAM-xl-A and PNIPAM$\mathrm{xl}-\mathrm{C}$. The overall volume of water per unit mass of polymer $\left(\mathrm{v}_{\mathrm{w} 1}\right)$ requires additionally the knowledge of the density of the dry polymer ( $\rho_{\text {dry }}$; Eqn. 2 and cf. below).

$H_{\mathrm{v}}=\left(\frac{D_{\text {wet }}}{D_{\text {dry }}}\right)^{3}=\frac{1}{1-\phi_{\mathrm{w}}}$

$\mathrm{v}_{\mathrm{w} 1}=\frac{D_{\mathrm{wet}}^{3}-D_{\mathrm{dry}}^{3}}{\rho_{\mathrm{dry}} \times D_{\mathrm{dry}}^{3}}$.

Ultracentrifugation relies on a given relation between volume and mass of the moving particles as illustrated by the fundamental equation of this technique, which can be written in the form as given with Eq. 3:

$\frac{V_{\mathrm{sed}}}{C_{\mathrm{acc}}}=S=\frac{1}{18} \times \frac{1}{\eta_{0}} \times D_{\mathrm{mov}}^{2} \times\left(\rho_{\mathrm{p}}^{\mathrm{w}}-\rho_{0}\right)$.

In Eq. 3, $V_{\text {sed }}$ means the sedimentation velocity in $\mathrm{m} / \mathrm{s}$, $C_{\text {acc }}$ the centrifugational acceleration in $\mathrm{m} / \mathrm{s}^{2}, S$ the sedimentation coefficient in Svedberg $\left(1 S=10^{-13} \mathrm{~s}\right), \eta_{0}$ the viscosity of the continuous phase (water) in $\mathrm{kg} /(\mathrm{s} \mathrm{m}), D_{\text {mov }}$ the diameter of the moving particle in $\mathrm{nm}, \rho_{\mathrm{p}}^{\mathrm{w}}$ the density of
Fig. 4 Change of the average particle size with temperature measured with dynamic light scattering (filled symbols; intensity-weighted average diameter, $D_{\mathrm{i}}$ ) and AUC (open symbols); data were obtained under isothermal condition during AUC and after about 20-min equilibration time for the DLS measurements
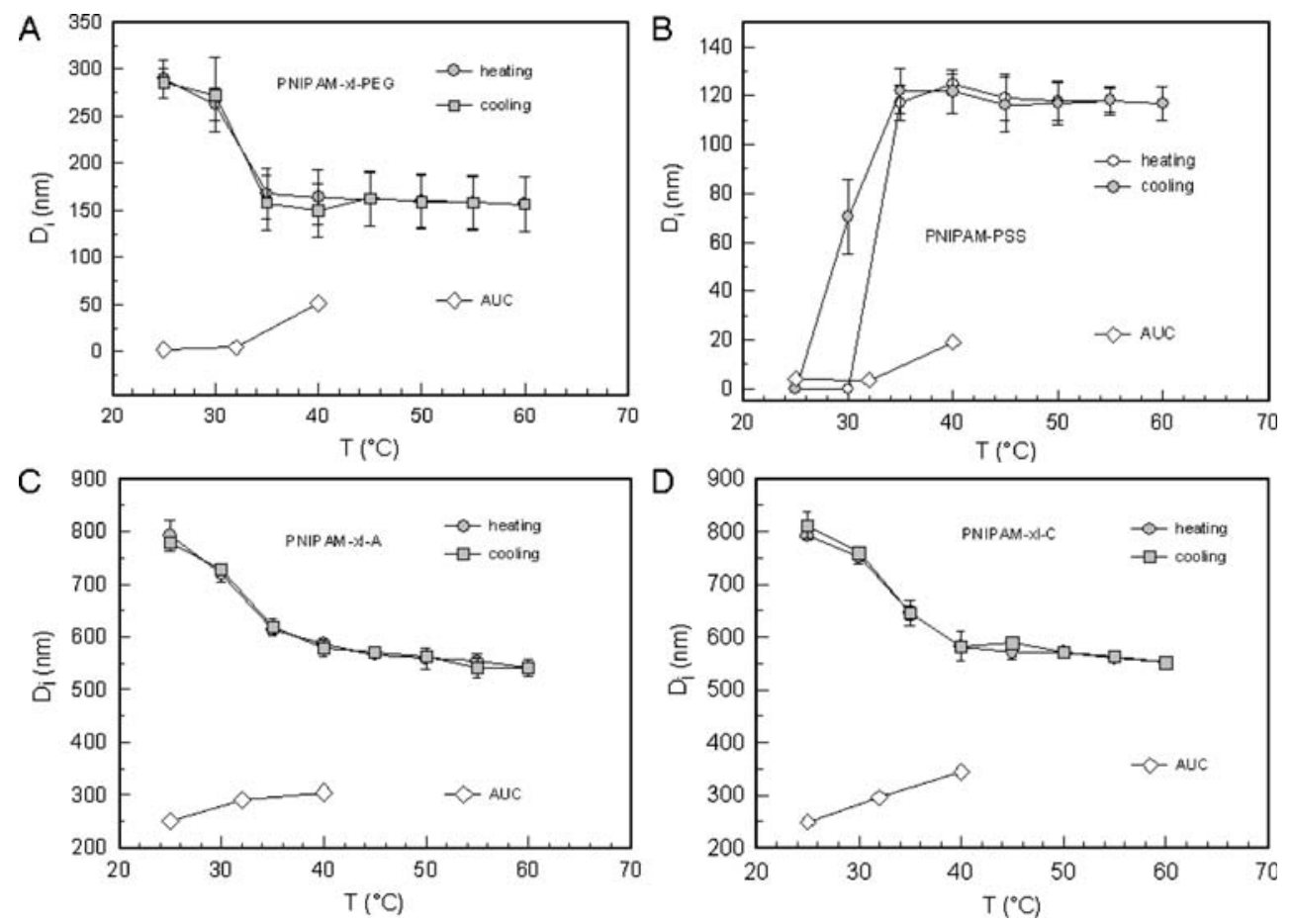
Table 2 Apparent diameter and density of the PNIPAM particles determined by AUC in $\mathrm{H}_{2} \mathrm{O}$ and $\mathrm{D}_{2} \mathrm{O}$ at different temperatures

\begin{tabular}{lcccccc}
\hline Sample & $D_{\text {mov }}(\mathrm{nm}) 25{ }^{\circ} \mathrm{C}$ & $\rho_{\mathrm{p}}^{\mathrm{W}}\left(\mathrm{g} / \mathrm{cm}^{3}\right) 25{ }^{\circ} \mathrm{C}$ & $D_{\text {mov }}(\mathrm{nm}) 32{ }^{\circ} \mathrm{C}$ & $\rho_{\mathrm{p}}^{\mathrm{W}}\left(\mathrm{g} / \mathrm{cm}^{3}\right) 32{ }^{\circ} \mathrm{C}$ & $D_{\text {mov }}(\mathrm{nm}) 40{ }^{\circ} \mathrm{C}$ & $\rho_{\mathrm{p}}^{\mathrm{W}}\left(\mathrm{g} / \mathrm{cm}^{3}\right) 40{ }^{\circ} \mathrm{C}$ \\
\hline PNIPAM-xl-PEG & 2.1 & 1.200 & 2.4 & 1.139 & 51.5 & 1.107 \\
PNIPAM-PSS & 4 & 1.320 & 3.4 & 1.246 & 19 & 1.929 \\
PNIPAM-xl-A & 251 & 1.187 & 291 & 1.167 & 305 & 1.152 \\
PNIPAM-xl-C & 249 & 1.194 & 295 & 1.168 & 344 & 1.172 \\
\hline
\end{tabular}

the polymer and $\rho_{0}$ the density of the continuous phase. The sedimentation coefficient is experimentally accessible as the centrifugational acceleration is known and the sedimentation velocity is measured. Equation 3 suggests that for particles with unknown density and size, like the PNIPAM particles studied, from AUC runs in two solvents with different density $\left(\mathrm{H}_{2} \mathrm{O}, \mathrm{D}_{2} \mathrm{O}\right)$ the unknowns $\left(D, \rho_{\mathrm{p}}^{\mathrm{w}}\right)$ can be determined. The data summarised in Table 2 show that the method, which is successful for solid hydrophobic particles, fails for the PNIPAM particles.

For the microgels, the average particle size increases with temperature, which is the opposite of the expected behaviour and also contradictory to the DLS results (cf. Fig. 1). Only for the PNIPAM-PSS did block copolymer AUC show increasing size with temperature, but the values are much smaller than that from DLS. The density $\left(\rho_{\mathrm{p}}^{\mathrm{w}}\right)$ shows no general trend in either direction. The straightforward explanation for these results is that various amounts of both solvent molecules $\left(\mathrm{H}_{2} \mathrm{O}\right.$ and $\left.\mathrm{D}_{2} \mathrm{O}\right)$ adhere strongly and move along with the polymer, and hence, the constancy of the volume-mass relation is violated. Note that the dependence of $T_{\mathrm{p}}$ on the composition of the continuous phase $\left(\mathrm{H}_{2} \mathrm{O}, \mathrm{D}_{2} \mathrm{O}\right)$ is, if so ever, of minor importance, as the highest temperature $\left(40{ }^{\circ} \mathrm{C}\right)$ is also above the LCST of PNIPAM in $\mathrm{D}_{2} \mathrm{O}$ that is about $2^{\circ}$ greater as in $\mathrm{H}_{2} \mathrm{O}[48,49]$.

However, a further evaluation of the sedimentation data is possible with the assumption that the hydrodynamic diameter from DLS corresponds to the size of the moving particles in the centrifugational field. Applying this assumption to Eq. 1, the density difference $\Delta \rho=\left(\rho_{\mathrm{p}}^{\mathrm{W}}-\rho_{0}\right)$ can be calculated. $\Delta \rho$ values calculated in this way show quite significant differences between the samples reflecting the molecular architecture in a reasonable manner (cf. Fig. 5a).

The $\Delta \rho$ values as displayed in Fig. 5a suggest that the density of the moving objects is not constant with temperature, which again is a hint to bound water moving along with the polymers. Another interesting result is the different temperature dependence of $\Delta \rho$ observed for the cross-linked samples (PNIPAM-xl-PEG, PNIPAM-xl-A, PNIPAM-xl-C) and the linear block copolymer PNIPAMPSS. For the microgels, $\Delta \rho$ increases with increasing temperature as expected during deswelling. Furthermore, these data show that the PNIPAM-xl-PEG particles are much less cross-linked than the other two samples as already concluded from the TEM images. In contrast, $\Delta \rho$ decreases for PNIPAM-PSS with increasing temperature, hinting that increasing amounts of water are bound in the physical PNIPAM network that is gradually built up.

As the density of water decreases with temperature, the density of the moving particles $\left(\rho_{\mathrm{p}}^{\mathrm{w}}\right)$ must not possess the same dependence as $\Delta \rho$. Note that the $\rho_{\mathrm{p}}^{\mathrm{w}}$ values calculated in this way differ from those obtained by the standard evaluation of the AUC data in $\mathrm{H}_{2} \mathrm{O}$ and $\mathrm{D}_{2} \mathrm{O}$ (Table 2). Surprisingly, the PNIPAM samples split into two groups,
Fig. 5 Dependence of the density difference and the density of the moving particles in dependence on temperature calculated from the sedimentation coefficients according to Eq. 1
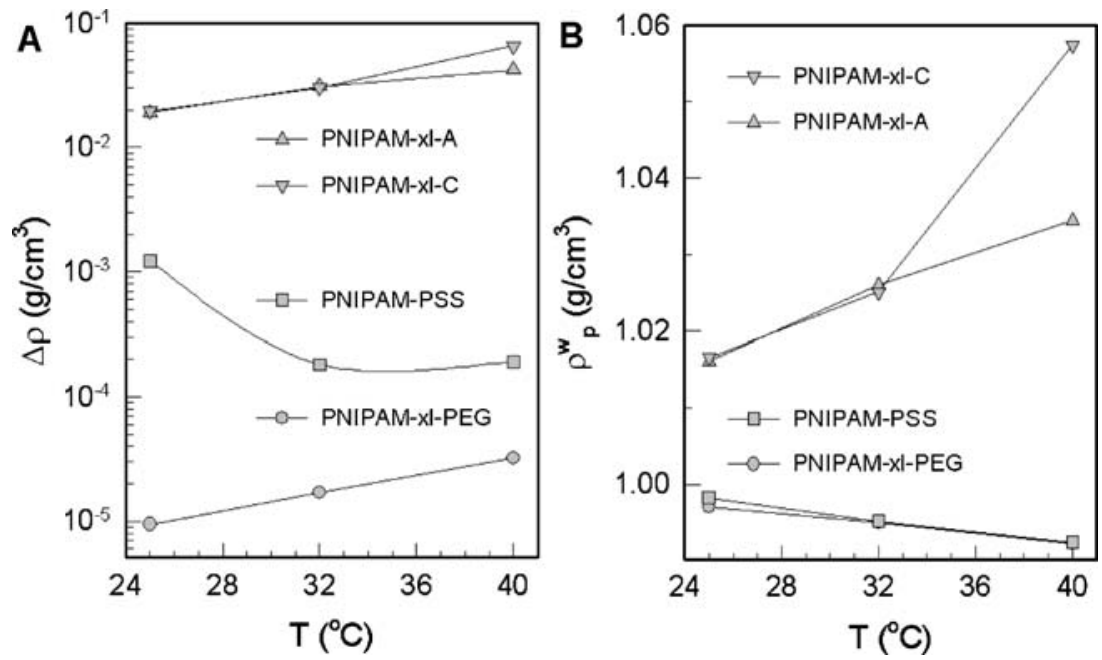
showing a distinctly different dependence of the $\rho_{\mathrm{p}}^{\mathrm{w}}$ values on temperature (Fig. 5b). The $\rho_{\mathrm{p}}^{\mathrm{w}}$ values for the cross-linked homopolymers (PNIPAM-xl-A and PNIPAM-xl-C) and the PNIPAM block copolymers (PNIPAM-xl-PEG and PNIPAMPSS) increase and decrease with increasing temperature, respectively. The homopolymers show the expected typical behaviour of PNIPAM, that is, deswelling and consequently increasing density with rising temperature. The contrary behaviour is observed for the block copolymers. The shrinkage of the PNIPAM core during heating and the exclusion of water are obviously overcompensated by the incorporation and binding of water molecules in the hydrophilic corona.

In order to further evaluate the $\Delta \rho$ values, the dry density of PNIPAM $\left(\rho_{\text {dry }}\right)$ must be known. The density of the microgel particles PNIPAM-xl-A and PNIPAM-xl-C was determined by ultracentrifugation in a density gradient built up in a methanol bromoform mixture. This study suggests a density for these particles between 1.35 and $1.42 \mathrm{~g} \cdot \mathrm{cm}^{-3}$, which is slightly higher than $1.269 \mathrm{~g} \cdot \mathrm{cm}^{-3}$ used in [50] for calculating the PNIPAM-water interaction by means of a lattice-fluid hydrogen-bond theory. Either of these values for the density of the dry PNIPAM is clearly larger than the values calculated from the $\Delta \rho$ values (cf. data of Fig. 5). The same holds also for the block copolymer samples, as the density of PEG and PSS are between 1.2 and $1.3 \mathrm{~g} \cdot \mathrm{cm}^{-3}[51,52]$. The density of the moving particles can be expressed by Eq. 4 leading to Eq. 5 for the volume of water bound per unit mass of monomer.

$\rho_{\mathrm{p}}^{\mathrm{w}}=\frac{m_{\mathrm{p}}}{v_{\mathrm{p}}+v_{\mathrm{bw}}}$

$\mathrm{v}_{\mathrm{w} 2}=\frac{\rho_{\mathrm{dry}}-\rho_{\mathrm{p}}^{\mathrm{w}}}{\rho_{\mathrm{p}}^{\mathrm{W}} \times \rho_{\mathrm{dry}}}$.
In order to calculate the amount of bound water as mol $\left(n_{\mathrm{bw}}\right)$ or mass $\left(m_{\mathrm{w} 2}\right)$ ratio, the density of the bound water must be known. As this value in dependence on temperature is not available, the bulk density of water can be used at least to get an idea about $n_{\mathrm{bw}}$ and $m_{\mathrm{w} 2}$. The $n_{\mathrm{bw}}$ and $m_{\mathrm{w} 2}$ values as depicted in Fig. $6 \mathrm{a}, \mathrm{b}$ are exactly in the same order of magnitude as those calculated in [50] and experimentally determined in [5]. These values correspond to the water molecules bound via hydrogen bonds to the NIPAM units.

The data of Fig. 7 compare the amount of overall water in the microgel particles $\left(\mathrm{v}_{\mathrm{w} 1}\right)$ with the amount of bound water, which adheres so strongly that it moves along with the polymer in the centrifugational field $\left(\mathrm{v}_{\mathrm{w} 2}\right)$.

The data of Fig. 7 suggest that the cationic microgel particles swell to slightly larger extent than the anionic particles. In general, this might be due to several reasons, as the degree of swelling is mainly influenced by the degree of cross-linking, the particle size and the interfacial tension. As the particles size is almost identical (cf. Fig. 4c, d), differences in the cross-linking density and the interfacial tension remain as possible reasons. Moreover, there is ample experimental evidence that the structure of microgel particles is heterogeneous regarding the cross-linking density, that is, the cross-links are non-homogeneously distributed and their number decreases towards the shell $[53,54]$.

The amount of water in either type of microgel particles decreases over the whole temperature range, whereby the drop is the steepest in the vicinity of the LCST. At $40{ }^{\circ} \mathrm{C}$, the overall water volume fraction in the particles $\left(\phi_{\mathrm{w}}\right)$ is still about $42 \%$ and $51 \%$ for PNIPASM-xl-A and PNIPAMxl-C, respectively. A similar value has been found for PNIPAM microgels with carboxylic end groups [55]. The $\mathrm{V}_{\mathrm{w} 2}$ values at $60{ }^{\circ} \mathrm{C}$ correspond to $\phi_{\mathrm{w}}$ of even $26 \%$ (PNIPAM-xl-A) and 43\% (PNIPASM-xl-C). Moreover,
Fig. 6 Molar (a) and mass (b) ratio of the water bound to PNIPAM in the highly crosslinked microgel particles in dependence on temperature; $n_{\mathrm{bw}}$ is expressed as mole water per mole repeating unit
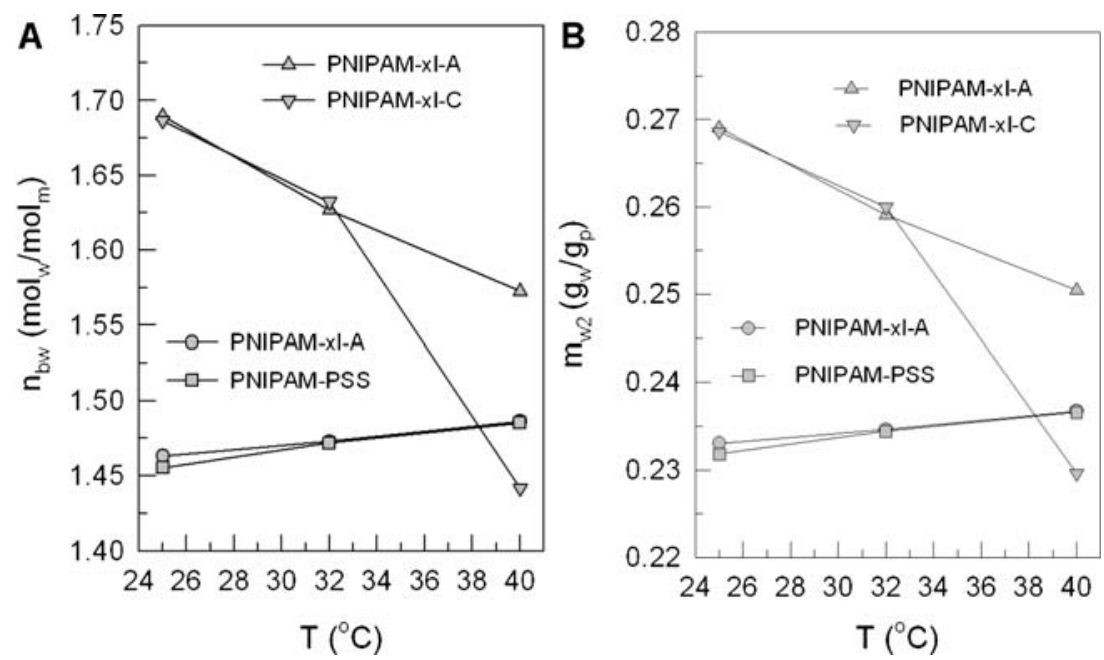


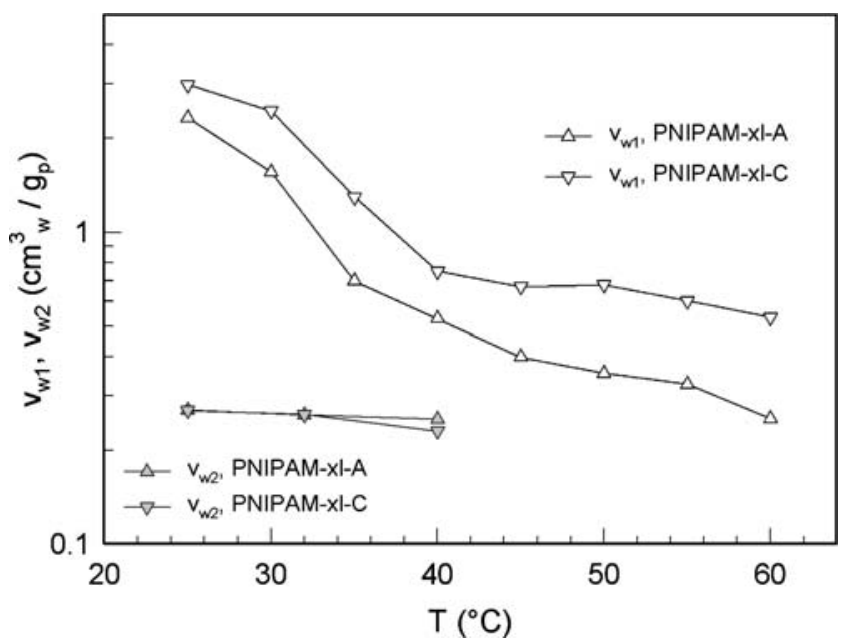

Fig. 7 Overall $\left(\mathrm{v}_{\mathrm{w} 1}\right)$ and bound water $\left(\mathrm{v}_{\mathrm{w} 2}\right)$ in the highly cross-linked microgel particles in dependence on temperature

the experimental data prove that PNIPAM microgels contain two types of water: free bulk water that easily drains the particles and bound water that strongly adheres to the polymer. The amount of the latter is practically independent of the nature of the ionic groups in the microgel and corresponds at $40{ }^{\circ} \mathrm{C}$ to a volume fraction of about $25 \%$. The fact that $\mathrm{v}_{\mathrm{w} 1}$ is over the whole range of temperature larger than $\mathrm{v}_{\mathrm{w} 2}$ proves that the combination of molecular characteristics determined with very different methods (electron microscopy, dynamic light scattering, analytical ultracentrifugation) leads to reliable conclusions. It must be mentioned that these results are in qualitative agreement with previous results obtained for coil-to-globule transition of a single PNIPAM chain with laser light scattering [47]. In addition, the evaluation of neutron scattering data for cross-linked PNIPAM particles revealed that at $25^{\circ} \mathrm{C}$ and in the shrunken state $\left(50^{\circ} \mathrm{C}\right)$, the polymer volume fraction is about 0.1 and 0.5 , respectively, depending on particular size and cross-linking density $[54,56]$.

\section{Zeta potential and charge density}

PNIPAM samples with charges increase and decrease their electrostatic surface potential during the heating and cooling cycles, respectively. The samples of this study differ distinctly in their surface properties as exemplarily illustrated by the zeta potential $(\zeta)$ at temperature above the LCST (cf. Fig. 8). The zeta potential spans a range of almost $100 \mathrm{mV}$ which is clearly related to the chemical nature of the samples. It is known that for soft particles, the zeta potential is less meaningful than the Donnan potential that is built up in the polyelectrolyte layer, and hence, a special data evaluation should be used [57]. However, for the sake of comparison between the different samples employed in this study, the applied relation to convert mobility in zeta potential seems to be sufficient. All samples, including the nominally electrically neutral PNIPAM-xl-PEG, possess a zeta potential. The slightly negative $\zeta$ of PNIPAM-xl-PEG is explainable with the assumption of a contact potential as described by Coehn's rule [58]. Accordingly, an electric potential exists between two materials with different permittivity in a way that the material with the lower dielectric constant carries the negative charge.

In dependence of the magnitude of the temperatureinduced size change, the zeta potential of the particles changes as well. The data of Fig. 9 illustrate the changes in dependence on the particle size and temperature. Figure $9 b$ relates the zeta potential to the particle surface and can be considered as measure, of course not exactly but as an estimate, of a surface potential. This value increases during heating as the average particle size decreases. From a colloid chemical point of view, this is good, as it counteracts instability which is usually enhanced at higher temperatures.

Expectedly, the size dependence is, especially for the PNIPAM-PSS sample, extremely steep (Fig. 9a). This is because firstly, the size changes only little above the LCST, and secondly, the stabilizing polyelectrolyte chains carry a huge number of charges compared with the other samples.

URT - a powerful tool to investigate thermal phase transitions of polymers

URT technology allows to evaluate either the ultrasound velocity or the ultrasound attenuation. The ultrasound velocity in fluids depends on the compressibility and the density of the medium. The attenuation is more complex and influenced by much more parameters (especially viscosity as well as vibration and structure relaxation processes) [59], and hence, it is charged with larger scatter. Therefore, it is more convenient to consider the difference

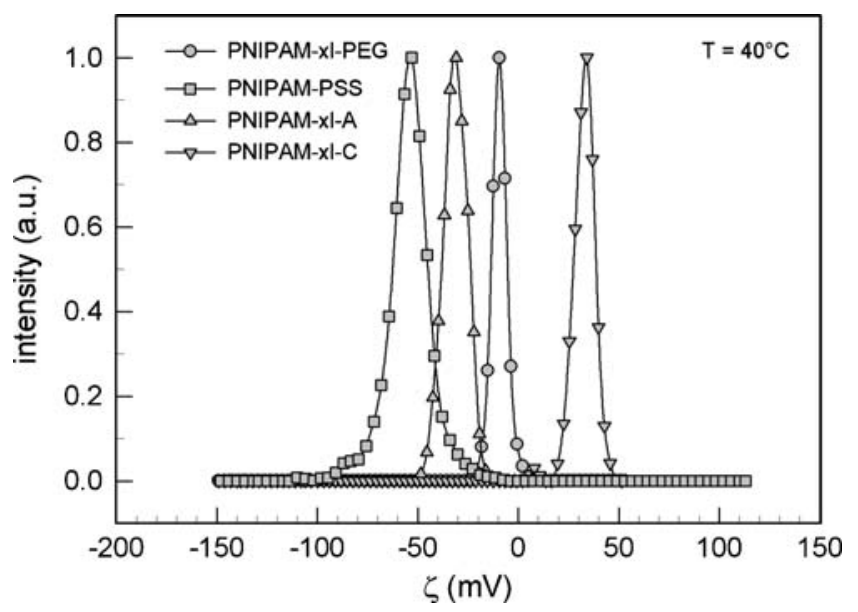

Fig. 8 Zeta potential $(\zeta)$ of the different PNIPAM samples at $40{ }^{\circ} \mathrm{C}$ 
Fig. 9 Zeta potential in dependence on the hydrodynamic particle size $\left(D_{\mathrm{i}}\right)(\mathbf{a})$ and zeta potential (absolute value) divided by the squared hydrodynamic particle size in dependence on temperature (b); the difference in the particle size for the PNIPAM-PSS in comparison to the data of Fig. 4 is due to the fact that for the block copolymer, the particle size above $T_{\mathrm{p}}$ depends on the concentration [59], and for the measurements considered here, the concentration is about eight times higher (0.12 instead of 0.014 wt.\%)
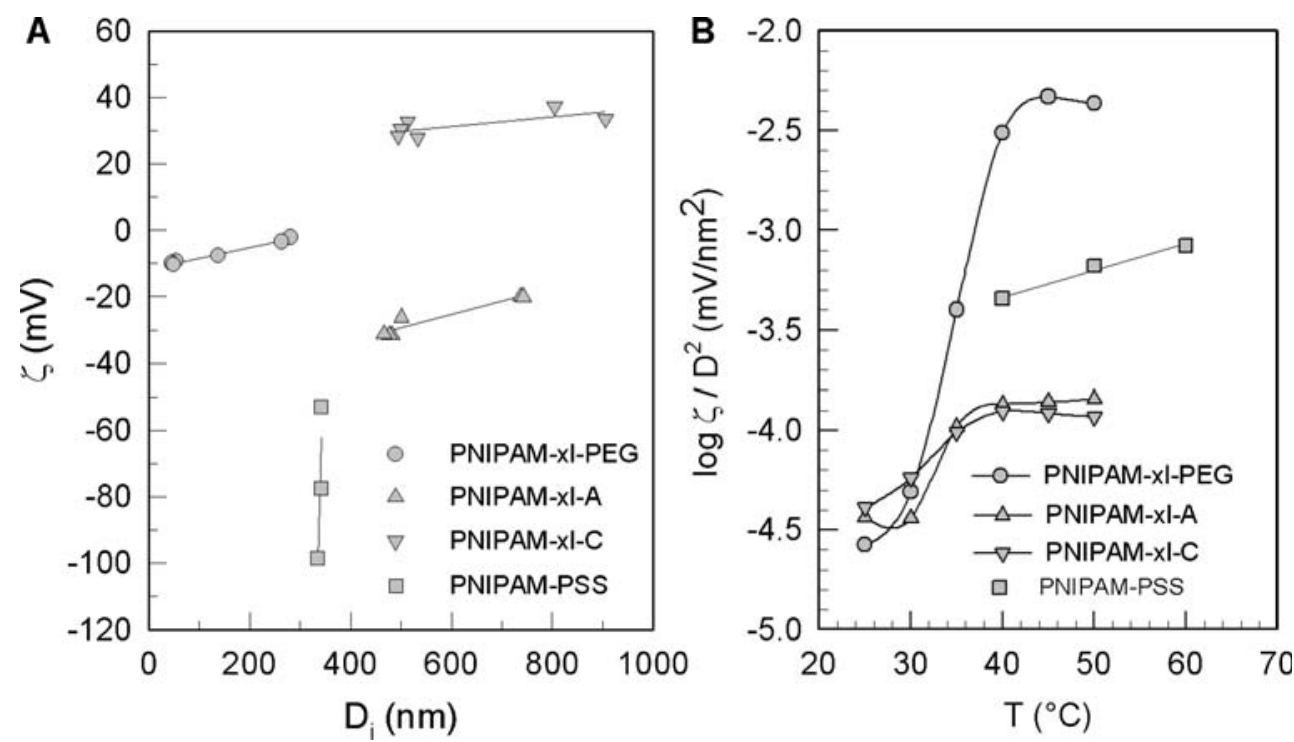

of the ultrasound velocity especially when diluted samples are investigated.

URT principally differs from light scattering or other optical techniques, as it does not rely on alteration of the turbidity but on the propagation of sound waves that is sensitive to both changes in the physical state of the polymer and the solvent molecules. This analytical technique is considered to belong to the methods with an extremely high resolution in measurements of physical parameters of solutions and colloidal suspensions [59]. Thus, the method seems to be ideally suited to investigate the critical solution behaviour of polymers. Very recently, the apparent activation free energies of the precipitation and redissolution of PNIPAM-PSS block polymers have been determined for the first time by means of transient-thermal studies with URT [60].

Figure 10 elucidates by means of runs with sample PNIPAM-xl-C the kind of data that are obtained with URT.

The curves of $\Delta A$ and $\Delta U$ exhibit completely different shapes and contain also different information. The $\Delta U-$ temperature curve is characterised by just one point of inflection (U1 at T1) which gives the LCST of the PNIPAM block (cf. also [60]). At this temperature, the coil-globule transition of the PNIPAM block takes place and the formerly homogeneous solution changes to a more or less turbid dispersion. On the contrary, the $\Delta A-T$ curves show at least three characteristic points (A1, A2, A3) during both heating and cooling. The point of inflection (A1) occurs again in the range of the LCST, whereas T2 and T3 at A2 (sharp maximum in the $\Delta A-T$ curve) and A3 (shallow minimum in the $\Delta A-T$ curve), respectively, appear only in the curves of the ultrasound attenuation at temperatures above the LCST. Moreover, A2 and A3 are only observed for the microgel particles (cf. Fig. 11) and not for the PNIPAM-PSS block copolymer.
The great difference in $\Delta A$ between the samples can be obviously related rather to the morphology of the samples than to the nature of the charge or the charge density. The order of $\Delta A$ reflects the density of the PNIPAM core, as it is the lowest for the non-cross-linked block copolymer and the highest for the heavily cross-linked microgel particles. Moreover, these data allow the conclusion that the reason for the transitions at $\mathrm{T} 2$ and $\mathrm{T} 3$ is connected with transitions in the cross-linked PNIPAM core, whereas T1 is clearly caused by the change in the solubility of the PNIPAM moieties.

In contrast to the $\Delta A-T$ curves (Fig. 11), the $\Delta U-T$ dependencies show for all curves a similar feature that is a steep minimum at the LCST in the $\mathrm{d} \Delta U / \mathrm{d} T$ curves (Fig. 12).

The $\mathrm{d} \Delta U / \mathrm{d} T$ curves as depicted in Fig. 12 reveal a hysteresis between the heating and cooling curves that also

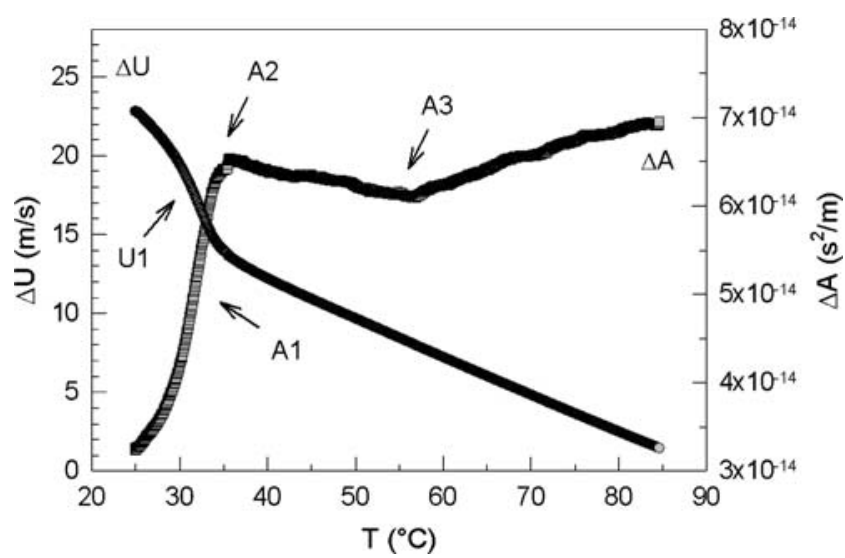

Fig. 10 Change of $\Delta U$ and $\Delta A$ during temperature scans between $25{ }^{\circ} \mathrm{C}$ and $85{ }^{\circ} \mathrm{C}$ for sample PNIPAM-xl-C with heating rate of $300 \mathrm{mK} / \mathrm{min}$; the arrows point to characteristic changes that have been considered in the further data evaluation (cf. text) 
Fig. 11 Change of the difference of the ultrasound attenuation $(\Delta A)$ during heating and cooling in the temperature range between $25^{\circ} \mathrm{C}$ and $85^{\circ} \mathrm{C}$ for all samples (a) and magnification of the $\Delta A-T$ curves for the charged microgel particles PNIPAM-xl-A and PNIPAMxl-C (b) (the order of legends corresponds to the order of the curves); the curves are averages over three or five consecutive runs; heating/cooling rate $300 \mathrm{mK} / \mathrm{min}$

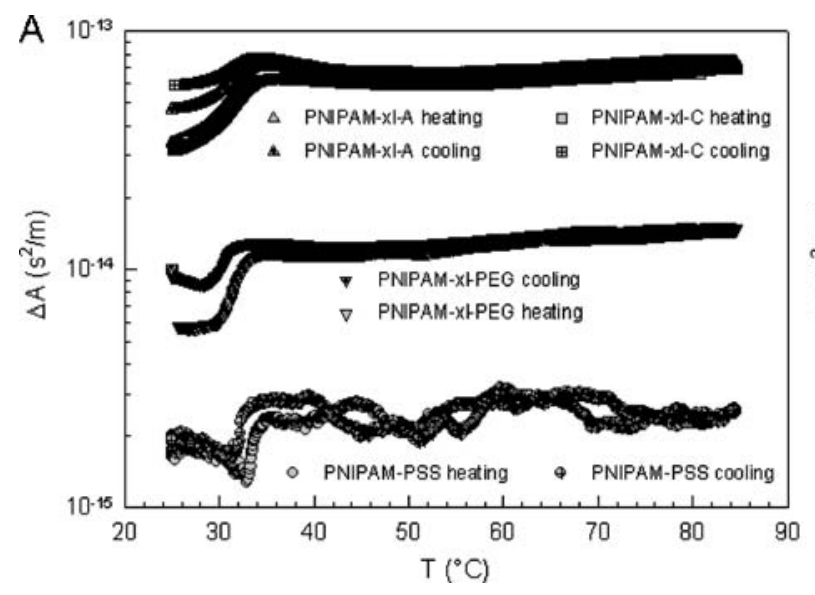

strongly depends on the morphology of the sample. For the highly cross-linked samples, the hysteresis, expressed as difference of the temperature between heating and cooling extremum, is below $0.3^{\circ}$ but increases for the loosely crosslinked PNIPAM-xl-PEG sample to $0.55^{\circ}$ and to $0.99^{\circ}$ for the block copolymer. Note that the magnitude of the hysteresis depends on the heating and cooling rate as detailed in [60]. Moreover, the graphs of Fig. 12 show that also the width of the transition range depends on the crosslinking density of the samples. The peak is broad for the microgel particles PNIPAM-xl-A and PNIPAM-xl-C and almost spike-like for the block copolymer.

Figure 13 includes a summary of the transition temperatures detected with $\Delta A$ and $\Delta U$ for all samples. This collection elucidates nicely the influence of the both the composition of the sample and the nature of the particular ultrasound property used for the analysis.

Besides the chemical nature of the samples and the degree of cross-linking, the charge density of the particles/ molecules can be related to the transition temperatures as
Fig. 12 Temperature derivatives of $\Delta U-T$ curves for the four PNIPAM samples during heating and cooling in the temperature range between $25^{\circ} \mathrm{C}$ and $45^{\circ} \mathrm{C}$; each graph shows three repeats; heating/cooling rate $300 \mathrm{mK} / \mathrm{min}$
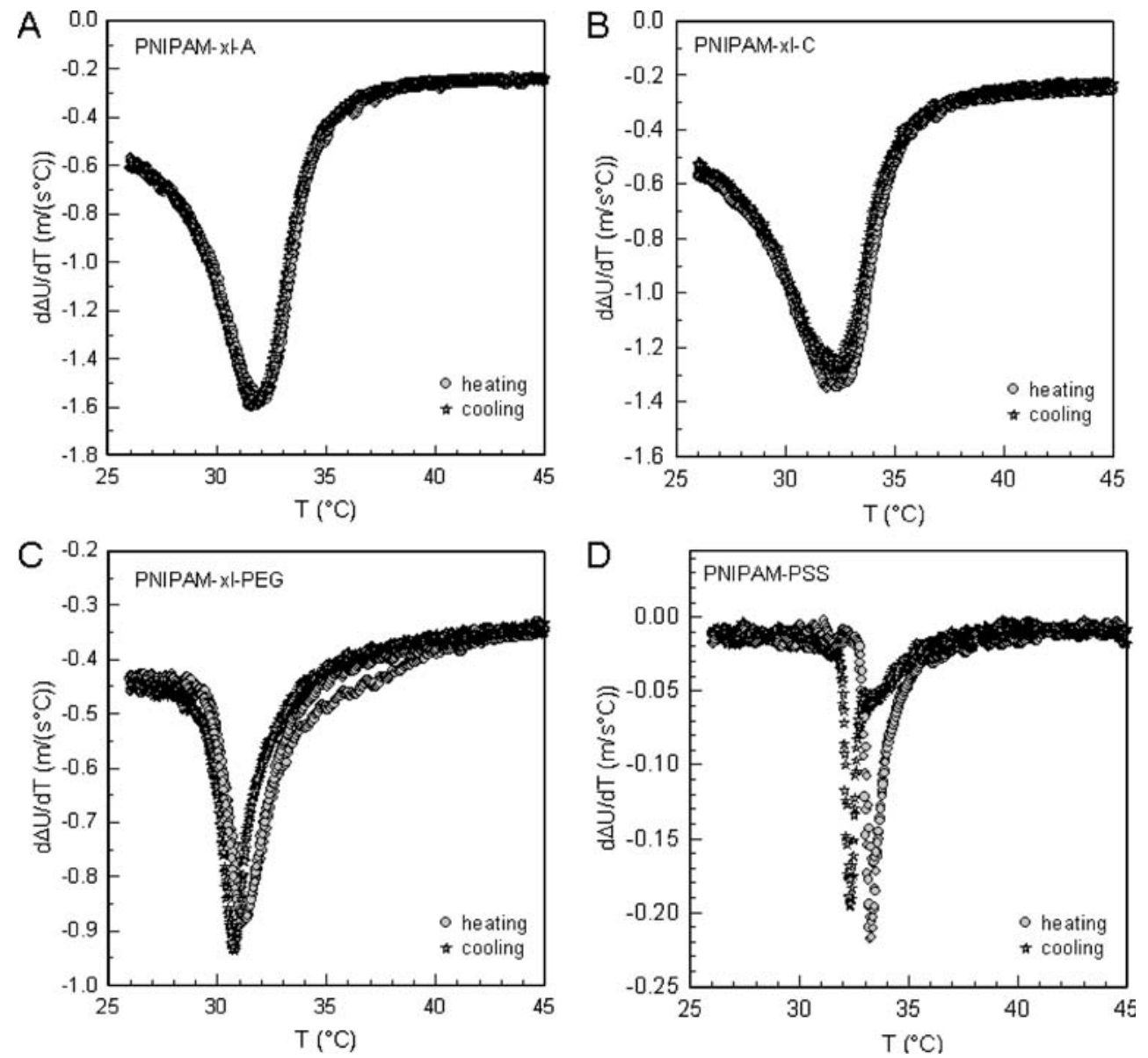

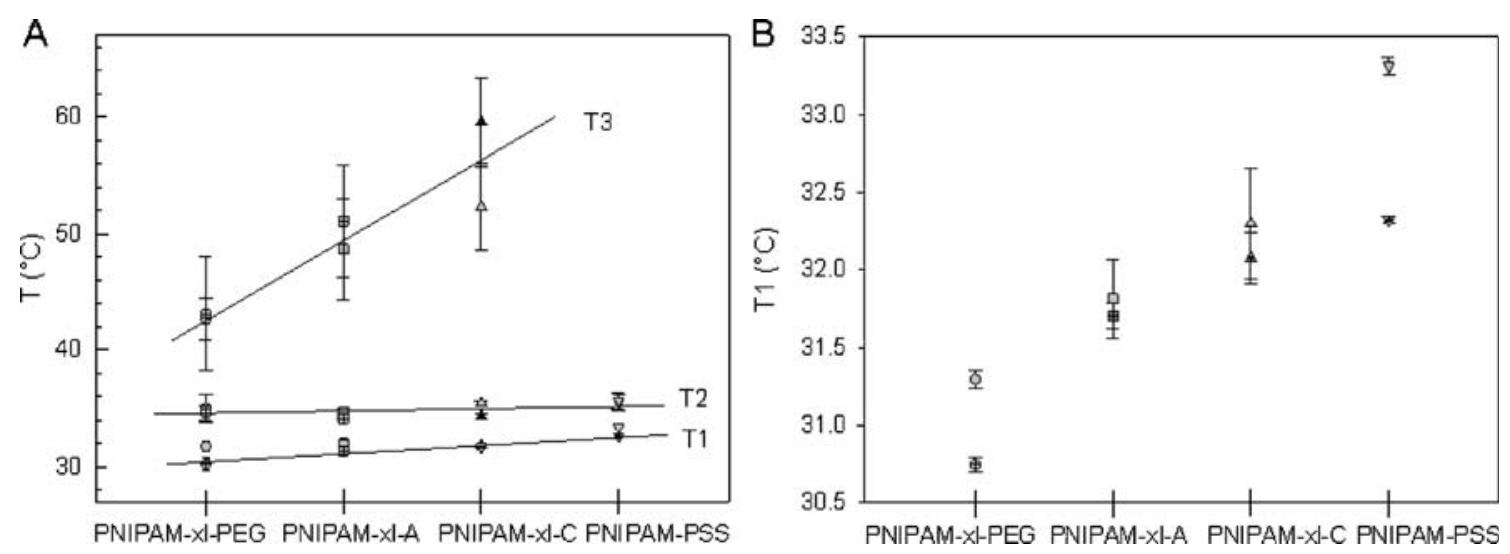

Fig. 13 Transition temperatures for the different PNIPAM samples determined from $\Delta A-T$ curves (a) and $\Delta U-T$ curves (b); the plain grey and crossed grey symbols represent heating and cooling runs, respectively, the lines in a are just for guiding the eye

well. Figure 14 shows that with increasing zeta potential, the first transition temperature (T1), that is, the LCST, increases for both the precipitation during heating and the dissolution during cooling. This result seems, at least for the precipitation, absolutely reasonable, as covalently attached charges counteract the aggregation of the PNIPAM moieties. This counterforce should be the stronger the higher the charge density. Therefore, the value of $\mathrm{T} 1$, determined from the difference of the ultrasound velocity, varies for the precipitation of $2^{\circ}$ between the electrically neutral PNIPAM-xl-PEG and the highly charged PNIPAMPSS block copolymers. However, there must be still other effects acting, as the largest difference of about $2.38^{\circ}$ is measured for $\mathrm{T} 1$ during cooling in the $\Delta A$ curves. The reason might that the PNIPAM-PSS block copolymer molecules must completely disentangle during the dissolution, whereas the microgel particles only expand.

The URT technology is indeed a powerful tool to investigate phase transitions. The data presented here show not only one characteristic temperature (T1) but also another two characteristic transitions for microgel particles at higher temperatures if the attenuation of the ultrasound is evaluated. Unfortunately, these changes cannot unambiguously be directly connected with structural changes either inside the cross-linked cores of the microgel particles or the hydrophilic shells or the water structure in either of these phases.

\section{Conclusions}

The results of this comprehensive study of four chemically different PNIPAM samples clearly show that the combination of methods leads to new insights in the thermoreversible properties of this polymer. The combination TEM, DLS and AUC allows for microgel particles to distinguish between the amount of tightly bound water and the quantity of water that 'only' swells the microgels. The evaluation of data obtained with DLS, zeta potential
Fig. 14 Dependence of the LCST (T1) on the absolute value of the zeta potential: $\mathbf{a}$ and b show $\mathrm{T} 1$ values for heating and cooling, respectively; the values of the zeta potential at $50{ }^{\circ} \mathrm{C}$
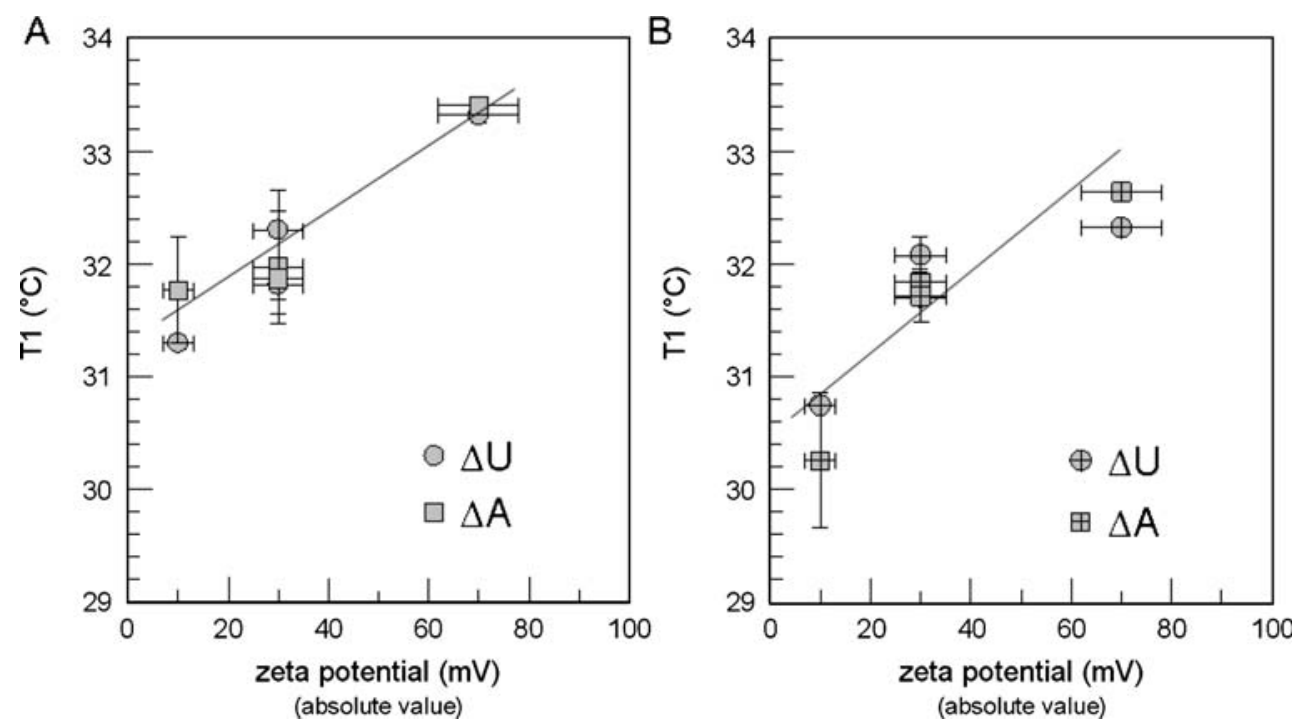
measurements and URT permits the identification of other characteristic transition temperatures above the LCST which are only hardly, if so ever, accessible by optical methods alone. By the evaluation of the ultrasound attenuation, two more transitions at temperatures above the LCST have been observed for PNIPAM microgel particles, but not for the PNIPAM-PSS block copolymer. The joint evaluation of this data with measurements of the electrophoretic mobility revealed a strong relation between the zeta potential of the precipitated structures and the transition temperatures for both the precipitation (during heating) and the redissolution (during cooling).

Acknowledgement The authors thank Mrs. Ursula Lubahn and Mrs. Sylvia Pirok for preparative and analytical assistance and Mrs. Rona Pitschke and Mrs. Heike Runge for the electron microscopy images. Financial support of the Max Planck Society and the TF Instruments $\mathrm{GmbH}$ is gratefully acknowledged. The interest of Theodor Funk in the progress of this work and many discussions with him are appreciated.

Open Access This article is distributed under the terms of the Creative Commons Attribution Noncommercial License which permits any noncommercial use, distribution, and reproduction in any medium, provided the original author(s) and source are credited.

\section{References}

1. Heskins M, Guillet JE (1968) J Macromol Sci Chem 2:1441

2. Eliassaf J (1978) J Appl. Polym Sci 22:873

3. Pelton RH, Chibante P (1986) Colloids Surf 20:247

4. Bae YH, Okano T, Hsu R, Kim SW (1987) Makromol Chem Rapid Commun 8:481

5. Dong LC, Hoffman AS (1990) J Control Release 13:21

6. Hoffman AS (1987) J Control Release 6:297

7. Hoffman AS, Afrassiabi A, Dong LC (1986) J Control Release 4:213

8. Coughlan DC, Quilty FP, Corrigan OI (2004) J Control Release 98:97

9. Aseyev VO, Tenhu H, Winnik FM (2006) Adv Polym Sci 196:1

10. Matsuoka H, Fujimoto K, Kawaguchi H (1998) Polym Gels Netw 6:319

11. Meunier F, Elaissari A, Pichot C (1995) Polym Adv Technol 6:489

12. Saunders B, Vincent B (2006) Responsive microgel dispersions. In: Somasundaran P (ed) Encyclopedia of surface and colloid science, vol 7. Taylor \& Francis, New York, p 5430

13. Saunders BR, Crowther HM, Morris GE, Mears SJ, Cosgrove T, Vincent B (1999) Colloids Surf A: Physicochem Eng Aspects 149:57

14. Saunders BR, Vincent B (1999) Adv Colloid Interface Sci 80:1

15. Meunier F, Elaissari A (2003) Surf Sci Ser 115:117

16. Afroze F, Nies E, Berghmans H (2000) J Mol Struct 554:55

17. Van Durme K, Van Assche G, Aseyev V, Raula J, Tenhu H, Van Mele B (2007) Macromolecules 40:3765

18. Bao LY, Zha LS (2006) J Macromol Sci A: Pure Appl Chem 43:1765

19. Bulmus V, Ding ZL, Long CJ, Stayton PS, Hoffman AS (2000) Bioconjugate Chem 11:78

20. Chen GH, Hoffman AS (1995) Nature 373:49

21. Dong LC, Hoffman AS (1991) J Control Release 15:141
22. Furyk S, Zhang YJ, Ortiz-Acosta D, Cremer PS, Bergbreiter DE (2006) J Polym Sci A: Polym Chem 44:1492

23. Schilli CM, Zhang MF, Rizzardo E, Thang SH, Chong YK, Edwards K, Karlsson G, Muller AHE (2004) Macromolecules 37:7861

24. Tauer K, Mukhamedjanova M, Holtze C, Nazaran P, Lee J (2007) Macromol Symp 248:227

25. Singh N, Lyon LA (2008) Colloid Polym Sci 286:1061

26. Blackburn WH, Lyon LA (2008) Colloid Polym Sci 286:563

27. Chung JE, Yokoyama M, Aoyagi T, Sakurai Y, Okano T (1998) Adv Colloid Interface Sci 53:119

28. Chung JE, Yokoyama M, Suzuki K, Aoyagi T, Sakurai Y, Okano T (1997) Colloids Surf B Biointerfaces 9:37

29. Ding ZL, Chen GH, Hoffman AS (1996) Bioconjugate Chem 7:121

30. Lopez-Leon T, Elaissari A, Ortega-Vinuesa JL, Bastos-Gonzalez D (2007) Chem Phys Chem 8:148

31. Lopez-Leon T, Ortega-Vinuesa Juan L, Bastos-Gonzalez D, Elaissari A (2006) J Chem Phys B 110:4629

32. Ricka J, Meewes M, Nyffenegger R, Binkert T (1990) Phys Rev lett $65: 657$

33. Takei YG, Aoki T, Sanui K, Ogata N, Okano T, Sakurai Y (1993) Bioconjugate Chem 4:42

34. Yamazaki A, Song JM, Winnik FM, Brash JL (1998) Macromolecules 31:109

35. Keerl M, Richtering W (2007) Colloid Polym Sci 285:471

36. Suzuki D, Kawaguchi H (2006) Colloid Polym Sci 284:1443

37. Zhang JT, Liu XL, Fahr A, Jandt KD (2008) Colloid Polym Sci 286:1209

38. Meewes M, Ricka J, Desilva M, Nyffenegger R, Binkert T (1991) Macromolecules 24:5811

39. Sata N, Saito S (1952) Koll Z Z Polymere 128:154

40. Brown PH, Schuck P (2006) Biophys J 90:4651

41. Schuck P, Rossmanith P (2000) Biopolymers 54:328

42. Schuck P (2000) Biophys J 78:1606

43. Lechner MD (1997) Macromol Rapid Commun 18:781

44. Tauer K, Khrenov V (2002) Macromol Symp 179:27

45. Topp MDC, Leunen IH, Dijkstra PJ, Tauer K, Schellenberg C, Feijen J (2000) Macromolecules 33:4986

46. Plantenberg T, Koetz J (2001) Polymer 42:3523

47. Wang XH, Qiu XP, Wu C (1998) Macromolecules 31:2972

48. Kujawa P, Winnik FM (2001) Macromolecules 34:4130

49. Wang XH, Wu C (1999) Macromolecules 32:4299

50. Lele AK, Hirve MM, Badiger MV, Mashelkar RA (1997) Macromolecules 30:157

51. Aranda P, Chen WJ, Martin CR (1995) J Membr Sci 99:185

52. Clinton N, Matlock P (1986) 1,2-Epoxide polymers. In: Kroschwitz JI (ed) Encyclopedia polymer science and engineering, vol 6 . Wiley, New York, p 225

53. Guillermo A, Addad JPC, Bazile JP, Duracher D, Elaissari A, Pichot C (2000) J Polym Sci B: Polym Phys 38:889

54. Stieger M, Richtering W, Pedersen JS, Lindner P (2004) J Chem Phys 120:6197

55. Saunders BR (2004) Langmuir 20:3925

56. Berndt I, Pedersen JS, Lindner P, Richtering W (2006) Langmuir 22:459

57. Ohshima H (2007) Colloid Polym Sci 285:1411

58. Coehn A (1898) Ann Phys 64:217

59. Buckin V, Smyth C (1999) Sem Food Anal 4:113

60. Tauer K, Gau D, Schulze S, Hernandez H (2008) Polymer 49:5452 